\title{
DISCOVERY OF HE 1523-0901, A STRONGLY $r$-PROCESS-ENHANCED METAL-POOR STAR WITH DETECTED URANIUM ${ }^{1}$
}

\author{
Anna Frebel, ${ }^{2,3}$ Norbert Christlieb, ${ }^{4,5}$ John E. Norris, ${ }^{2}$ Christopher Thom, ${ }^{6,7}$ \\ Timothy C. BeERs, ${ }^{8}$ AND JAEHyon RheE ${ }^{9,10}$ \\ Received 2006 November 21; accepted 2007 March 15; published 2007 April 11
}

\begin{abstract}
We present age estimates for the newly discovered, very $r$-process-enhanced metal-poor star HE 1523-0901 $([\mathrm{Fe} / \mathrm{H}]=-2.95)$ based on the radioactive decay of Th and $\mathrm{U}$. The bright $(V=11.1)$ giant was found among a sample of bright metal-poor stars selected from the Hamburg/ESO Survey. From an abundance analysis of a high-resolution $(R=75,000)$ VLT/UVES spectrum, we find HE 1523-0901 to be strongly overabundant in $r$ process elements $([r / \mathrm{Fe}]=1.8)$. The abundances of heavy neutron-capture elements $(Z>56)$ measured in $\mathrm{HE}$ 1523-0901 match the scaled solar $r$-process pattern extremely well. We detect the strongest optical U line at $3859.57 \AA$. For the first time, we are able to employ several different chronometers, such as the U/Th, U/Ir, Th/ $\mathrm{Eu}$, and $\mathrm{Th} / \mathrm{Os}$ ratios to measure the age of a star. The weighted average age of HE 1523-0901 is $13.2 \mathrm{Gyr}$.
\end{abstract} Several sources of uncertainties are assessed in detail.

Subject headings: early universe - Galaxy: halo — nuclear reactions, nucleosynthesis, abundances stars: abundances - stars: individual (HE 1523-0901)

\section{INTRODUCTION}

The Galactic chemical history can be reconstructed by studying the most metal-poor stars. Of particular importance are direct age measurements of the oldest halo objects. Such ages can be inferred by comparing the observed abundance ratios of the radioactive elements ${ }^{232} \mathrm{Th}$ (half-life of $14 \mathrm{Gyr}$ ) and ${ }^{238} \mathrm{U}$ (4.5 Gyr) with theoretical predictions of their initial production. In 2001, Cayrel et al. (2001) announced the first detection of $\mathrm{U}$ in an $r$-process-enhanced metal-poor star, CS 31082-001 $([\mathrm{Fe} / \mathrm{H}]=-2.9)$. Together with a Th measurement, this led to the novel use of the U/Th ratio as a chronometer. This technique yielded an age of $14 \pm 3$ Gyr (Hill et al. 2002), in good agreement with the recent result of $13.7 \pm 0.2$ Gyr for the age of the universe, as determined from WMAP data (Spergel et al. 2006).

The similarities of the neighboring nuclei Th $(Z=90)$ and $\mathrm{U}(Z=92)$ cause this cosmic clock to be much less prone to systematic uncertainties than the more widely used $\mathrm{Th} / \mathrm{Eu}$ ( $Z=63$ for $\mathrm{Eu}$ ) chronometer (Goriely \& Clerbaux 1999; Schatz et al. 2002). Hence, it provides more reliable age estimates. It is thus important to attempt a $\mathrm{U}$ measurement in

\footnotetext{
${ }^{1}$ Based on observations made with ESO telescopes at the La Silla Paranal Observatory under program IDs 275.D-5028 and 077.D-0453.

${ }^{2}$ Research School of Astronomy and Astrophysics, Australian National University, Cotter Road, Weston, ACT 2611, Australia; jen@mso.anu.edu.au.

${ }^{3}$ McDonald Observatory and Department of Astronomy, University of Texas at Austin, 1 University Station, C1400, Austin, TX 78712; anna@ astro.as.utexas.edu.

${ }^{4}$ Department of Astronomy and Space Physics, Uppsala University, Box 515, SE-751-20 Uppsala, Sweden; norbert@astro.uu.se.

${ }^{5}$ Hamburger Sternwarte, Universität Hamburg, Gojenbergsweg 112, D21029 Hamburg, Germany.

${ }^{6}$ Department of Astronomy and Astrophysics, University of Chicago, Chicago, IL 60637; cthom@oddjob.uchicago.edu.

${ }^{7}$ Centre for Astrophysics and Supercomputing, Swinburne University of Technology, Mail 31, P.O. Box 218, Hawthorn, VIC 3122, Australia.

${ }^{8}$ Department of Physics and Astronomy, CSCE (Center for the Study of Cosmic Evolution), and JINA (Joint Institute for Nuclear Astrophysics), Michigan State University, East Lansing, MI 48824-1116; beers@pa.msu.edu.

${ }^{9}$ Center for Space Astrophysics, Yonsei University, Seoul 120-749, Korea.

${ }^{10}$ Space Astrophysics Laboratory, California Institute of Technology, MC 405-47, Pasadena, CA 91125; rhee@srl.caltech.edu.
}

more metal-poor stars. The strongest $U$ line at optical wavelengths is very weak, requiring very high quality spectroscopic data. In addition, it is located in the wing of a strong Fe line and is blended with a $\mathrm{CN}$ feature. Hence, low $\mathrm{C}$ and $\mathrm{N}$ abundances are vital for an accurate $\mathrm{U}$ abundance determination. It is thus not possible to measure $\mathrm{U}$ in C-rich $r$-process-rich stars such as CS 22892-052 (Sneden et al. 1996), for which only the $\mathrm{Th} / \mathrm{Eu}$ chronometer can be employed. These observational difficulties explain why, currently, CS 31082-001 is the only known strongly $r$-process-enhanced metal-poor star with a reliably measured U abundance (Cayrel et al. 2001; Hill et al. 2002). However, one more object is known to exhibit U (V. Hill et al. 2007, in preparation), while for another one, at least a tentative detection has been made (Cowan et al. 2002).

Here we report the discovery of a strongly $r$-process-enhanced star with a $U$ measurement. This detection, in combination with other neutron-capture abundances, makes possible stellar nucleochronometry. The giant HE 1523-0901 ( $V=$ 11.1) was found in a sample of bright metal-poor stars (Frebel et al. 2006) from the Hamburg/ESO Survey (HES). Based on an initial metallicity estimate of $[\mathrm{Fe} / \mathrm{H}]=-2.7$, obtained from a medium-resolution (FWHM $\sim 2 \AA$ ) spectrum, the star was observed with high spectral resolution $(R \sim 45,000)$ with MIKE at the Clay Magellan telescope. The spectrum revealed a strong enhancement of heavy neutron-capture elements, and a tentative detection of $\mathrm{U}$ was made.

\section{OBSERVATIONS}

To confirm the tentative U detection in HE 1523-0901, new observations were obtained with the Ultraviolet-Visual Echelle Spectrograph (UVES; Dekker et al. 2000) at the ESO Very Large Telescope (VLT). The star was observed in service mode in 2005 August and 2006 April. We made use of the image slicer No. 2 and a $0.45^{\prime \prime}$ slit width to achieve a very high resolving power of $R \sim 75,000$. We employed the BLUE $346 \mathrm{~nm}$ setting covering 3050-3874 $\AA$ and the BLUE $437 \mathrm{~nm}$ setting covering 3758$4990 \AA$. The two settings conveniently overlap at $3860 \AA$ where the $\mathrm{U}$ line is located. The total exposure time was $7.5 \mathrm{hr}$, of which $5 \mathrm{hr}$ were spent with the BLUE $346 \mathrm{~nm}$ and $2.5 \mathrm{hr}$ with the BLUE $437 \mathrm{~nm}$ setting. 
We use the pipeline-reduced spectra provided by the ESO Data Management and Operations Division. The frames, shifted to the stellar rest frame, are co-added. We estimate a signalto-noise ratio of $\mathrm{S} / \mathrm{N} \sim 350$ per $12.4 \mathrm{~m} \AA$ pixel at $\sim 3900 \AA$, the region where the two settings overlap.

\section{ABUNDANCE ANALYSIS}

For our one-dimensional LTE abundance analysis of the VLT/UVES spectrum, we use the latest version of the MARCS code (B. Gustafsson et al. 2007, in preparation). Solar abundances are taken from Asplund et al. (2005). For the choice of atomic absorption lines of the lighter elements, we use a line list based on the compilations of Barklem et al. (2005) and Aoki et al. (2002), as well as our own collection retrieved from the VALD database (Kupka et al. 1999). The line list provided in Hill et al. (2002) was updated with the latest atomic data used for the measurements of the neutron-capture elements. The molecular line data for CH (B. Plez 2006, private communication) are based on $g f$-values and line positions from LIFBASE (Luque \& Crosley 1999), excitation energies are taken from Jørgensen et al. (1996), and isotopic shifts are computed by Plez. The CN line data (B. Plez 2006, private communication) is described in Hill et al. (2002).

Using the Alonso et al. (1999) calibration, we determine an effective temperature of $T_{\text {eff }}=4630 \pm 40 \mathrm{~K}$ (random error) from dereddened BVRI CCD photometry (Beers et al. 2007) and JHK 2MASS data (Skrutskie et al. 2006). The microturbulence was obtained by demanding no trend of elemental abundances with equivalent width. Using Fe I lines, the result is $v_{\text {micr }}=2.6 \pm 0.3 \mathrm{~km} \mathrm{~s}^{-1}$. From the LTE Fe I-Fe II ionization equilibrium, we derive a surface gravity of $\log g=1.0 \pm$ 0.3 . The resulting metallicity is $[\mathrm{Fe} / \mathrm{H}]=-2.95 \pm 0.2$ for $\mathrm{HE}$ $1523-0901$.

Equivalent widths are obtained by fitting Gaussian profiles to the chosen atomic lines. For blended lines and molecular features, we use the spectrum synthesis approach, in which the abundance is obtained by matching the observed to a synthetic spectrum of known abundance. This technique is extensively used for the Th II line region around $4019 \AA$, the U II line region around $3860 \AA$, and other important line regions (e.g., $\mathrm{Eu}$, Ir, and Os). Abundance uncertainties arising from this method are usually driven by difficulties with the continuum placements and vary from 0.05 to 0.15 dex.

\section{NEUTRON-CAPTURE ELEMENTS}

The spectrum of HE 1523-0901 shows numerous strong lines of $\sim 25$ neutron-capture elements associated with the $r$ process. In Figure 1, the observed abundance pattern of the star is shown. Error bars represent the standard error of the mean abundance of several lines for each element. As can be seen, it closely follows the scaled $([r / \mathrm{Fe}]=1.8)$ solar $r$-process pattern of Burris et al. (2000). For neutron-capture elements with $56<Z<77$, the agreement is excellent (the standard error of the mean is 0.02 ; see bottom panel of Fig. 1).

Several Th II lines are detected. However, most of them are severely blended with lines from other elements. We regard the line at $4019.13 \AA$ as the most reliable one because the contaminating blends are best known from the literature. First, a strong ${ }^{13} \mathrm{CH}$ feature is located blueward $(4019.01 \AA)$ of the Th line. Despite the star having a ${ }^{12} \mathrm{C} /{ }^{13} \mathrm{C}$ ratio of $\sim 3-4$ (based on features at $\sim 4020, \sim 4220$, and $\sim 4310 \AA$ ), the total carbon abundance is subsolar, $[\mathrm{C} / \mathrm{Fe}]=-0.3$, and thus not a major contaminator. Second, the blending with the Ce II line at $4019.06 \AA$ can easily

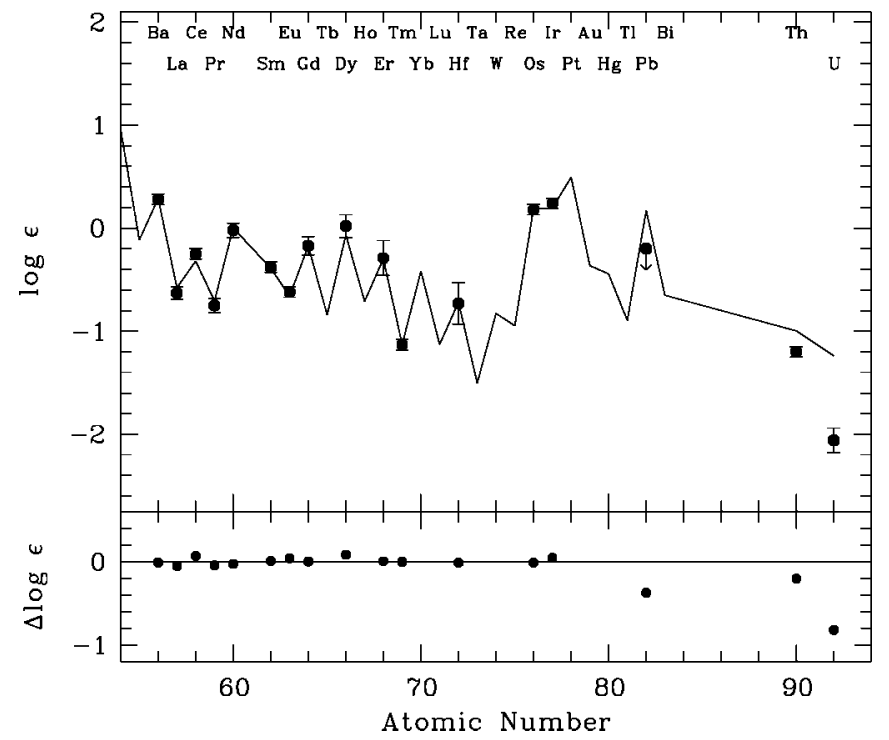

FIG. 1.-Neutron-capture element abundances of HE 1523-0901 in comparison with those from the solar $r$-process (Burris et al. 2000) scaled to match the observed elements with $56 \leq Z \leq 69$ (top panel). The bottom panel shows the residuals from the abundances of HE 1523-0901 minus the solar $r$-process values.

be accounted for by fitting the blue wing of the observed feature. Fortunately, the red wing is dominated by the Th line and allows for a well-constrained fit. Any additional contamination of the whole region with numerous Co I lines is not significant.

Other strong Th II lines are located in bluer regions of the spectrum with lower S/N. Also, the line fits suffer from unidentified features and blends that are difficult to account for. Due to these problems, we adopt the abundance of $\log \epsilon(\mathrm{Th})=-1.20$ from the line Th II $\lambda 4019$.

In our high-resolution, high $\mathrm{S} / \mathrm{N}$ spectrum of HE 15230901, we detect the U II line at $3859.6 \AA$. The spectral region around this line is shown in Figure 2. We repeat here that low $\mathrm{C}$ and $\mathrm{N}$ abundances are crucial for a $\mathrm{U}$ detection because it is significantly blended with a $\mathrm{CN}$ feature. Both the $\mathrm{U}$ line and the $\mathrm{CN}$ feature, in turn, are located in the wing of a very strong, saturated $\mathrm{Fe}$ I line. This complicates the fitting procedure. To obtain a best fit of this region, ${ }^{11}$ we increase the VALD $\log g f$ value of the Fe I line at 3859.911 by 0.24 dex. The C $([\mathrm{C} / \mathrm{Fe}]=-0.15)$ and $\mathrm{N}([\mathrm{N} / \mathrm{Fe}]=0.6)$ abundances employed in the $\mathrm{U}$ synthesis are well constrained by a good fit to the violet $\mathrm{CN}$ feature at $3883 \AA$. The $\mathrm{U}$ abundance is then obtained by simultaneously fitting the $\mathrm{Fe}, \mathrm{CN}$, and $\mathrm{U}$ lines. We derive an abundance of $\log \epsilon(\mathrm{U})=-2.06$. The final $\mathrm{U} / \mathrm{Th}$ ratio for HE $1523-0901$ is $\log \epsilon(\mathrm{U} / \mathrm{Th})=-0.86$. Further abundance ratios of radioactive to naturally occurring $r$-process elements are listed in Table 1.

The abundances of Eu, Os, and $\mathrm{Ir}$ are $\log \epsilon(\mathrm{Eu})=-0.62$, $\log \epsilon(\mathrm{Os})=0.18$, and $\log \epsilon(\mathrm{Ir})=0.24$. We also attempted to detect $\mathrm{Pb}$, the decay product of $\mathrm{Th}$ and $\mathrm{U}$. However, it could not be detected in the current spectrum of HE 1523-0901 (the $\mathrm{S} / \mathrm{N}$ in this region is $\sim 150)$. As shown in Figure 1, the upper limit of $\log \epsilon(\mathrm{Pb})<-0.2$ is below the expected abundance of the scaled $r$-process pattern. A full discussion of the complete

\footnotetext{
${ }^{11}$ We note that the two features in the red wing of the Fe I line at $3859.6 \AA$ (see Fig. 2) were previously unidentified (Hill et al. 2002). Based on the new Sm atomic data (Lawler et al. 2006), we find the bluer feature (3860.28 $\AA$ ) to be a Sm line. The other feature remains unidentified.
} 

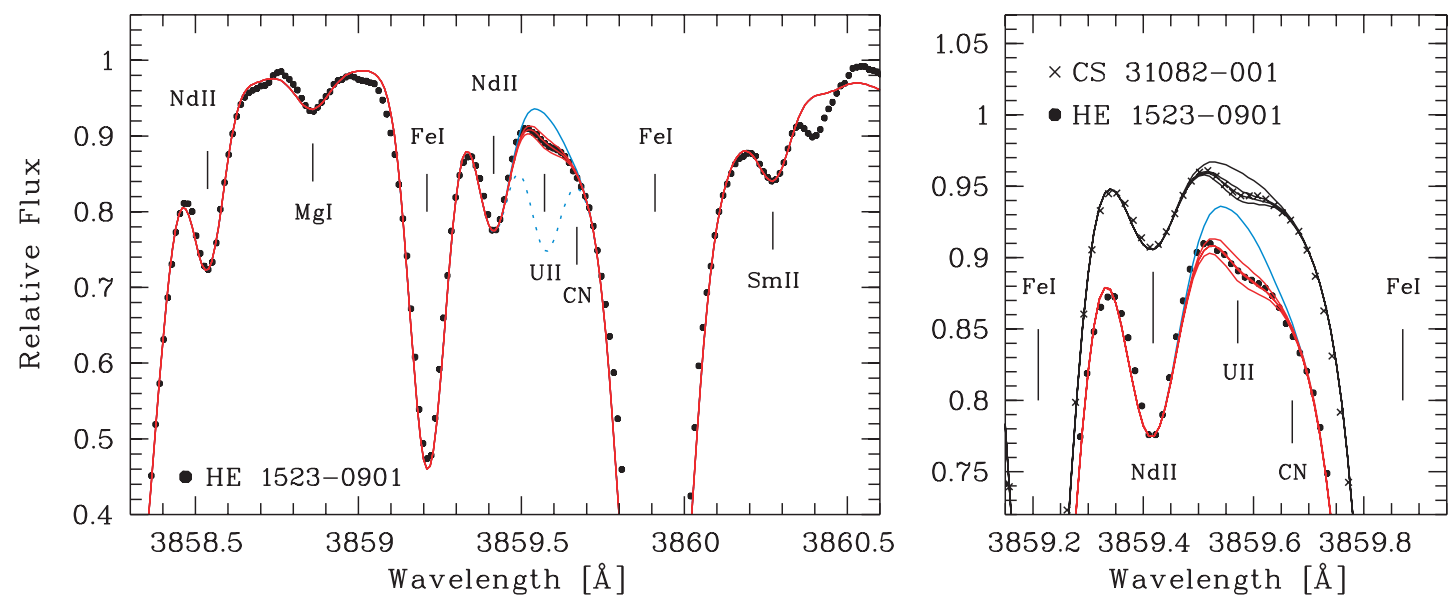

FIG. 2.-Spectral region around the U II line in HE 1523-0901 (filled circles) and CS 31082-001 (crosses; right panel only). Overplotted are synthetic spectra with different $\mathrm{U}$ abundances of $\log \epsilon(\mathrm{U})=$ none, $-1.96,-2.06$, and -2.16 (HE 1523-0901) and $\log \epsilon(\mathrm{U})=$ none, $-2.05,-2.15$, and -2.25 (CS 31082-001). The dotted line in the left panel corresponds to a scaled solar $r$-process $U$ abundance present in the star if no $U$ had decayed. Positions of other features are indicated.

abundance analysis will be given elsewhere (A. Frebel et al. 2007 , in preparation).

To test our derived abundances, we measured Th II $\lambda 4019$ and the U features in the spectrum of CS 31082-001 that was used by Hill et al. (2002). Figure 2 shows the U region for CS 31082-001 (crosses). Despite differences in the employed model atmospheres, we obtain a $\log \epsilon(\mathrm{U} / \mathrm{Th})$ ratio of -0.93 for CS 31082-001. This is in very good agreement with the published value of -0.89 , as derived from these two lines.

We estimate a fitting uncertainty of 0.05 dex for the Eu, Os, $\mathrm{Ir}$, and $\mathrm{Th}$ abundances. The $\mathrm{U}$ abundance is driven by the fit of the $\mathrm{Fe}$ line close to the $\mathrm{U}$ line. Changing the $\mathrm{C}$ abundance by +0.1 dex results in only a -0.02 dex different $\mathrm{U}$ abundance. Changing the $\mathrm{Fe}$ abundance by +0.1 dex changes the $\mathrm{U}$ abundance by -0.12 . We adopt a 0.12 dex uncertainty for $U$.

\section{NUCLEOCHRONOMETRY}

There are three types of chronometers that involve the abundances of Th, $\mathrm{U}$, and naturally occurring $r$-process elements (Cayrel et al. 2001). The subscript "initial" refers to the initial

TABLE 1

Ages Derived from Different Abundance Ratios

\begin{tabular}{clccrc}
\hline \hline $\mathrm{X} / \mathrm{Y}$ & $\log (\mathrm{PR})^{\mathrm{a}}$ & Ref. & $\log \epsilon(\mathrm{X} / \mathrm{Y})_{\text {obs }}$ & $\begin{array}{c}\text { Age } \\
(\mathrm{Gyr})\end{array}$ & $\begin{array}{c}\text { Uncertainties }^{\mathrm{b}} \\
(\mathrm{Gyr})\end{array}$ \\
\hline $\mathrm{Th} / \mathrm{Eu} \ldots \ldots$ & -0.377 & 1 & -0.58 & 9.5 & $3.3 / 3.4 / 0.6 / 0.6 / 5.6$ \\
& -0.33 & 2 & -0.58 & 11.7 & $3.3 / 3.3 / 0.5 / 0.5 / 5.6$ \\
& -0.295 & 3 & -0.58 & 13.3 & $3.3 / 3.0 / 0.2 / 0.2 / 5.6$ \\
$\mathrm{Th} / \mathrm{Os} \ldots \ldots$. & -1.15 & 2 & -1.38 & 10.7 & $3.3 / 2.8 / 5.6 / 0.0 / 5.6$ \\
$\mathrm{Th} / \mathrm{Ir} \ldots \ldots$. & -1.18 & 2 & -1.44 & 12.1 & $3.3 / 1.9 / 2.8 / 1.4 / 5.6$ \\
& -1.058 & 1 & -1.44 & 17.8 & $3.3 / 2.0 / 2.9 / 1.5 / 5.6$ \\
$\mathrm{U} / \mathrm{Eu} \ldots \ldots$. & -0.55 & 2 & -1.44 & 13.2 & $1.9 / 0.6 / 0.4 / 0.2 / 1.6$ \\
$\mathrm{U} / \mathrm{Os} \ldots \ldots$. & -1.37 & 2 & -2.24 & 12.9 & $1.9 / 0.6 / 1.2 / 0.3 / 1.6$ \\
$\mathrm{U} / \mathrm{Ir} \ldots \ldots \ldots$ & -1.40 & 2 & -2.30 & 13.3 & $1.9 / 0.3 / 0.3 / 0.7 / 1.6$ \\
& -1.298 & 3 & -2.30 & 14.8 & $1.9 / 0.3 / 0.3 / 0.8 / 1.6$ \\
$\mathrm{U} / \mathrm{Th} \ldots \ldots .$. & -0.301 & 4 & -0.86 & 12.2 & $2.8 / 0.4 / 0.9 / 0.4 / 2.2$ \\
& -0.29 & 5 & -0.86 & 12.4 & $2.8 / 0.4 / 0.9 / 0.4 / 2.2$ \\
& -0.256 & 3 & -0.86 & 13.1 & $2.8 / 0.5 / 1.0 / 0.5 / 2.2$ \\
& -0.243 & 6 & -0.86 & 13.4 & $2.8 / 0.4 / 0.8 / 0.4 / 2.2$ \\
& -0.22 & 2 & -0.86 & 13.9 & $2.8 / 0.4 / 0.9 / 0.4 / 2.2$ \\
\hline
\end{tabular}

ReFERENCES. - (1) Sneden et al. 2003; (2) Schatz et al. 2002; (3) Cowan et al. 2002; (4) Goriely \& Arnould 2001; (5) Wanajo et al. 2002; (6) Dauphas 2005.

${ }^{a}$ Initial production ratio.

${ }^{b}$ Age uncertainties arising from uncertainties in observed measurements/ $T_{\text {eff }} / \log g / v_{\text {micr }} / \mathrm{PR}$. production ratio (PR), while the subscript "now" refers to the observed value:

$$
\begin{aligned}
& \text { 1. } \Delta t=46.7\left[\log (\mathrm{Th} / r)_{\text {initial }}-\log \epsilon(\mathrm{Th} / r)_{\text {now }}\right] ; \\
& \text { 2. } \Delta t=14.8\left[\log (\mathrm{U} / r)_{\text {initial }}-\log \epsilon(\mathrm{U} / r)_{\text {now }}\right] ; \\
& \text { 3. } \Delta t=21.8\left[\log (\mathrm{U} / \mathrm{Th})_{\text {initial }}-\log \epsilon(\mathrm{U} / \mathrm{Th})_{\text {now }}\right] \text {. }
\end{aligned}
$$

Using several different chronometers and PRs, we derive a set of ages for HE 1523-0901. The results are given in Table 1. Where available, we list several PRs for each chronometer to illustrate the available range and the subsequent spread in the derived ages. We take the weighted average of all the individual ages to derive a final age of 13.2 Gyr for HE $1523-0910$.

Forming an average based on weights obtained from the uncorrelated observational uncertainties is an arbitrary choice that only minimizes the observational (statistical) uncertainties but not necessarily the systematic uncertainties. Using different weights, for example by omitting the $\mathrm{Th} / \mathrm{r}$ ratios, would lead to slightly larger observational, but smaller systematic, uncertainties. A weighted observational uncertainty in the abundance ratios arising from the fitting procedure results in an $0.7 \mathrm{Gyr}$ weighted uncertainty for the final age. This value is driven by the uncertainty of the uranium abundance measurement.

We also investigate the influence of variations of model atmosphere parameters $\left(T_{\text {eff }}, \log g, v_{\text {micr }}\right)$ on the stellar age. Adding these three age uncertainties in quadrature yields a $1.5 \mathrm{Gyr}$ weighted uncertainty in the final age. Any correlations of the different chronometers are thus automatically taken into account. To obtain an age uncertainty arising from the uncertainties in the PRs, we calculate $\sigma t=\sum_{i}\left(w_{i} \sigma t_{i}\right) / \sum_{i} w_{i}$ (with $w_{i}=1 / \sigma_{i}^{2}$, where $\sigma t_{i}$ is the age uncertainty from the different PRs and $\sigma_{i}$ the one from the observational uncertainty) as an upper bound, assuming the worst possible correlation(s) of the uncertainties in the PRs. We thus derive a 2.7 Gyr weighted uncertainty in the final age. For the calculation of the PR uncertainties, we followed Schatz et al. (2002), who list overall systematic uncertainties for all three types of chronometers. In Table 1, we list the five age uncertainties for all chronometers.

Due to the much shorter half-life of $\mathrm{U}$, uncertainties in ages derived from chronometers $\mathrm{U} / r$ are significantly smaller than for those derived from $\mathrm{Th} / r$. Excluding the $\mathrm{Th} / r$ chronometers yields a weighted average of 13.4 Gyr. The observational uncertainty then becomes $0.8 \mathrm{Gyr}$, and the combined model atmosphere uncertainty is 0.9 Gyr, while the PR uncertainty is much reduced to 
1.8 Gyr. This illustrates the superiority of the $\mathrm{U} / r$ ages in terms of systematic uncertainties. Also, $\sim 2$ Gyr is roughly the age spread caused by the different PRs for U/Th.

Another advantage of the special case of U/Th is that systematic observational uncertainties associated with model atmosphere parameters are less severe than for other ratios because the neighboring $\mathrm{U}$ and $\mathrm{Th}$ nuclides have very similar atomic parameters. It follows that the U/Th PR should be less sensitive to theoretical $r$-process model uncertainties compared with ratios of elements with larger mass separation such as the case of Th to Eu (Goriely \& Clerbaux 1999; Wanajo et al. 2002). However, in their extensive parameter study, Schatz et al. (2002) find very similar overall systematic uncertainties for the three chronometer types.

In order to derive reliable ages for individual metal-poor stars, it is crucial to have available stars with a very precise $\mathrm{U}$ measurements together with the most accurate theoretical PRs. Only then can nucleochronometry be refined to deliver the best possible Galactic stellar ages.

\section{DISCUSSION}

For the first time, it is possible to make use of all three types of chronometers in one star: $\mathrm{Th} / r, \mathrm{U} / r$, and $\mathrm{U} / \mathrm{Th}$. Previously, either the chronometer $\mathrm{Th} / r$ was employed (e.g., CS 22892052; Sneden et al. 1996) or only the ones based on the availability of U (CS 31082-001; Cayrel et al. 2001). Also, the U/ $r$ chronometers, such as $\mathrm{U} / \mathrm{Os}$ or $\mathrm{U} / \mathrm{Eu}$, for the first time deliver reasonable ages because, unlike CS 31082-001 (see Schatz et al. 2002), HE 1523-0901 appears to follow the solar $r$-process pattern from $\mathrm{Ba}$ all the way to U. Given the observational and theoretical uncertainties, the consistency of the individual ages derived for HE 1523-0901 is remarkable.

Since we also measure the U/Th ratio in CS 31082-001, it is possible to determine a relative age of the two stars. We find HE 1523-0901 to be 1.5 Gyr younger than CS 31082-001, which is independent of the employed production ratio. This age difference is based on only a 0.07 dex difference in the observed U/Th ratios. Given that the observational uncertainties exceed that ratio difference as well as the uncertainties associated with initial production ratios, the present ages of the two stars suggest that they formed at roughly the same time.
This is also reflected in their almost identical metallicity. We note that HE $1523-0901$ is $\sim 200 \mathrm{~K}$ cooler than CS 31082 001, which makes the lines in our star more prominent (see Fig. 2).

Stellar age measurements such as these provide a lower limit to the age of the Galaxy and, hence, the universe. Despite their large uncertainties, the age limits provided by HE 1523-0901 and CS 31082-001 are in good agreement with the WMAP result of $13.7 \mathrm{Gyr}$ for the age of the universe.

We note here that HE 1523-0901 is the first "uranium star" discovered in the HES. Only a very few $r$-process-enhanced stars are suitable for a detection of $U$ because the objects need to be bright, sufficiently cool, strongly overabundant in heavy neutron-capture elements, and have low $\mathrm{C}$ and $\mathrm{N}$ abundances. It is of great importance, however, to find further "uranium stars." This new group of objects will provide crucial observational constraints to the study of the $r$-process and its possible production site(s). They may also be used to empirically constrain the U/Th production ratio, which, in turn, may provide the much-needed feedback for theoretical works to improve the initial production ratios.

We thank the ESO staff for carrying out the VLT-UT2 observations and reducing the data. Allocation of VLT Director's Discretionary Time is gratefully acknowledged. We are indebted to V. Hill for providing a spectrum of CS 31082-001 and B. Plez for providing his newly compiled $\mathrm{CH}$ line list. Fruitful discussions on spectrum synthesis calculations with W. Hayek and C. Sneden are acknowledged. This research made extensive use of the Vienna Atomic Line Database (VALD). A. F. and J. E. N. acknowledge support from the Australian Research Council under grants DP0342613 and DP0663562. N. C. is a Research Fellow of the Royal Swedish Academy of Sciences supported by a grant from the Knut and Alice Wallenberg Foundation. He acknowledges support by Deutsche Forschungsgemeinschaft through grants Ch 214/3 and Re 353/ 44. T. C. B. is supported by the US National Science Foundation under grant AST 04-06784 as well as from grant PHY 0216783 (Physics Frontier Center/JINA).

\section{Facilities: VLT:Kueyen(UVES), Magellan:Clay(MIKE)}

\section{REFERENCES}

Alonso, A., Arribas, S., \& Martínez-Roger, C. 1999, A\&AS, 140, 261

Aoki, W., Norris, J. E., Ryan, S. G., Beers, T. C., \& Ando, H. 2002, PASJ, 54,933

Asplund, M., Grevesse, N., \& Sauval, A. J. 2005, in ASP Conf. Ser. 336, Cosmic Abundances as Records of Stellar Evolution and Nucleosynthesis, ed. T. G. Barnes III \& F. N. Bash (San Francisco: ASP), 25

Barklem, P. S., et al. 2005, A\&A, 439, 129

Beers, T. C., et al. 2007, ApJS, 168, 128

Burris, D. L., Pilachowski, C. A., Armandroff, T. E., Sneden, C., Cowan, J. J., \& Roe, H. 2000, ApJ, 544, 302

Cayrel, R., et al. 2001, Nature, 409, 691

Cowan, J. J., et al. 2002, ApJ, 572, 861

Dauphas, N. 2005, Nature, 435, 1203

Dekker, H., D’Odorico, S., Kaufer, A., Delabre, B., \& Kotzlowski, H. 2000, Proc. SPIE, 4008, 534

Frebel, A., et al. 2006, ApJ, 652, 1585

Goriely, S., \& Arnould, M. 2001, A\&A, 379, 1113
Goriely, S., \& Clerbaux, B. 1999, A\&A, 346, 798

Hill, V., et al. 2002, A\&A, 387, 560

Jørgensen, U. G., Larsson, M., Iwamae, A., \& Yu, B. 1996, A\&A, 315, 204

Kupka, F., Piskunov, N., Ryabchikova, T. A., Stempels, H. C., \& Weiss, W. W. 1999, A\&AS, 138, 119

Lawler, J. E., Den Hartog, E. A., Sneden, C., \& Cowan, J. J. 2006, ApJS, 162, 227

Luque, J., \& Crosley, D. R. 1999, SRI Int. Rep., MP 99-099

Schatz, H., Toenjes, R., Pfeiffer, B., Beers, T. C., Cowan, J. J., Hill, V., \& Kratz, K.-L. 2002, ApJ, 579, 626

Skrutskie, M. F., et al. 2006, AJ, 131, 1163

Sneden, C., McWilliam, A., Preston, G. W., Cowan, J. J., Burris, D. L., \& Amorsky, B. J. 1996, ApJ, 467, 819

Sneden, C., et al. 2003, ApJ, 591, 936

Spergel, D. N., et al. 2006, preprint (astro-ph/0603449)

Wanajo, S., Itoh, N., Ishimaru, Y., Nozawa, S., \& Beers, T. C. 2002, ApJ, 577, 853 